TMUP-HEL-9609

\title{
Difference Operator Approach to the Moyal Quantization and Its Application to Integrable Systems
}

\author{
Ryuji Kemmoku网 \\ Department of Physics, Tokyo Metropolitan University \\ Minami-Osawa, Hachioji, Tokyo 192-03, Japan
}

\begin{abstract}
Inspired by the fact that the Moyal quantization is related with nonlocal operation, I define a difference analogue of vector fields and rephrase quantum description on the phase space. Applying this prescription to the theory of the KP-hierarchy, I show that their integrability follows to the nature of their Wigner distribution. Furthermore the definition of the "expectation value" clarifies the relation between our approach and the Hamiltonian structure of the KPhierarchy. A trial of the explicit construction of the Moyal bracket structure in the integrable system is also made.
\end{abstract}

\footnotetext{
* to be published in J. Phys. Soc. Jpn.

**E-mail: kemmoku@phys.metro-u.ac.jp
} 


\section{Introduction}

In the classical mechanics, dynamics can be described in the geometrical context. If we define a Hamiltonian vector field on the phase space (more generally the symplectic manifold), the time evolution of canonical variables can be recognized as an integral curve of the Hamiltonian vector field. It is equivalent to a Lie derivative as an operation to a function defined in the phase space. In this sense the vector field generates a Lie algebra.

When we quantize some dynamical systems, we usually consider the Hilbert space instead of the phase space and replace the Poisson bracket of observables by a commutator of operators on the Hilbert space. This is nothing but Dirac's correspondence principle.

Meanwhile effort to realize quantization on the phase space has been also made. Weyl associated quantum mechanical operators to classical functions of position and momentum [1]. Wigner introduced the quantum "distribution function" (Wigner distribution) and formulated the quantum statistics on the phase space [2]. These two concepts were combined by Moyal [3]. He showed that if one constructs a classical function along Weyl's correspondence, an expectation value of it, which is given by using the Wigner distribution, can be identified with one of the corresponding quantum mechanical operator. On the basis of these works, many authors have contributed to the progress in formulation of the phase space quantization (e.g. see [4]- 10 ).

One of remarkable things of Moyal's work is the introduction of the so-called Moyal bracket. It plays a role of the commutator in the phase space quantization and reduces to the Poisson bracket in the classical limit. In the ordinary procedure the correspondence between the classical and quantum mechanics is somehow mysterious since there exists no explanation how such a transition takes place in the nature. But the phase space quantization seems to clarify such mechanism: As Moyal suggested in his paper, the Moyal bracket seems to be constructed by using some nonlocal operators. On the other hand, the uncertainty principle implies the existence of a minimal lattice size of the phase space. From these two facts we arrive at the speculation that some difference operator plays a key role in the Moyal quantization.

In Section 2, along this spirit, I first consider the difference analogue of the Hamiltonian vector field and represent the Moyal bracket by using it. This new vector field has a tricky property. The basis of it consists of the difference operator. For this reason a constant vector plays the role of indices which originally associate with the local coordinates. Then the dimension of the new vector field becomes infinite even if one of the phase space is finite as it will be seen. Moreover for the purpose of reformulating the Moyal quantization in our prescription, I regard the difference analogue of the Hamiltonian vector field as an operator on the phase space and define its dual form. The dual pairing provides us the measurement of the quantum mechanics in a form of expectation value in the phase space. I also discuss dynamics through the time evolution of the expectation value in a simple case. All these reproduce the well-known results in ordinary quantum mechanics. 
Although until now I mention only about the Moyal quantization, we already know other examples in which the difference version of a system plays an important role. One of them is nonlinear integrable systems. The existence of difference analogue of integrable systems is not trivial at all, since in general a naive discretization of continuous variables will not preserve integrability but creates chaos in an arbitrary nonlinear system. The transition between integrable and non-integrable discretizations is subtle and difficult to clarify the mechanism. It is, however, supposed that a large symmetry exists behind the integrable discrete system. We know, for instance, Hirota's bilinear difference equation [11], which is a difference version of the KP-hierarchy [12], exhibits large symmetry explicitly in an equivalent form of the Plücker relation [13]. For other types of integrable discretization, see, e.g., [14]. Besides these wellknown examples we have studied the role of the difference version of the integrable system. In [15], we clarified that the $W_{1+\infty}$ symmetry [16] in the KP theory is deeply connected with the difference analogue of a discrete version of the conformal symmetry. As mentioned above the difference analogue of Hamiltonian vector field has infinite dimensionality. This fact implies that we could apply such formulation to the integrable systems.

On the basis of the investigation, in Section 3, a main part of this manuscript, I try to apply the difference operator approach stated in Section 2 to the KP theory. There the phase space whose coordinates are regarded as the spectral parameter is considered. I first define a functional corresponding to the Wigner distribution by using the Baker-Akhiezer function in the place of the quantum wave function, and a quantity analogous to the expectation value in the Moyal quantization. We see that the "distribution" induces the KP solution via the additional symmetric flow [17, 18]. Furthermore the "expectation value" will suit the Hamiltonian structure of the KP-hierarchy [19]. At the end of this section, the Moyal bracket structure emerges.

Section 4 is devoted to construct the Moyal bracket explicitly in the integrable system. To this end I first pay attention to a fact that the algebraic structure of the new vector field is analogous to a discretization of the conformal symmetry. Applying it with the case of the $\mathrm{KdV}$ equation, I will provide a new bracket through the Moyal-like deformation of the Poisson structure of the KdV equation [20].

In the last section we summarize the results of this manuscript and provide future perspective.

Finally three appendices are given to supply details of our discussions. 


\section{Difference operator approach to the Moyal quantization}

In this section I explain the essence of the difference operator approach to the Moyal quantization based on the work in [21].

\subsection{Difference analogue of vector fields}

Let $M$ be a differentiable manifold $(\operatorname{dim} M=m)$. We take a local coordinate system $\vec{x}=\left(x_{1}, \cdots, x_{m}\right)$ on $M$ and define a (generalized) difference operator as

$$
\nabla_{\vec{a}}:=\frac{1}{\lambda} \sin \left(\lambda \sum_{j} a_{j} \partial_{x_{j}}\right)
$$

where $\vec{a}$ denotes a constant vector and $\lambda$ is a parameter. In the $\lambda \rightarrow 0$ limit, (2.1) becomes $\sum a_{j} \partial_{j}$. It is the ordinary vector field. This fact leads to the natural definition of a new vector field whose basis consists of $\nabla_{\vec{a}}$. I consider the following form:

$$
X^{\mathrm{D}}=\int d \vec{a} v_{\lambda}(\vec{x}, \vec{a}) \nabla_{\vec{a}},
$$

where $v_{\lambda}$ is the component of $X^{\mathrm{D}}$ in the local coordinate system $\vec{x}$ on $M$. Comparing with the ordinary vector fields of the differential geometry, we see that the constant vectors $\vec{a}$ play the role of the indices $j$ of the local coordinates $x_{j}$. In this sense, $X^{\mathrm{D}}$ is infinite-dimensional. From now we regard (2.2) as a difference analogue of the ordinary vector field.

Starting from (2.2), we can discuss the "difference geometry" by defining other geometrical objects:

Difference one-form $\Omega^{\mathrm{D}}$

$$
\Omega^{\mathrm{D}}:=\int d \vec{a} w_{\lambda}(\vec{x}, \vec{a}) \Delta^{\vec{a}},
$$

where $\Delta^{\vec{a}}$ is the conjugate of $\nabla_{\vec{a}}$ satisfying $\left\langle\Delta^{\vec{a}^{\prime}}, \nabla_{\vec{a}}\right\rangle=\delta\left(\vec{a}^{\prime}-\vec{a}\right)$. The one-form $\Delta^{\vec{a}}$ is, for example, realized as the pseudo-differential operator

$$
\lambda \csc \{\lambda(\vec{a} \cdot \vec{\partial})\}:=\frac{2 i \lambda}{e^{i \lambda \vec{a} \cdot \vec{\partial}}-e^{-i \lambda \vec{a} \cdot \vec{\partial}}}=2 i \lambda \sum_{n=0}^{\infty} e^{-i(2 n+1) \lambda \vec{a} \cdot \vec{\partial}} .
$$

This enables us to define the bilinear pairing explicitly:

$$
\left\langle\Delta^{\vec{a}^{\prime}}, \nabla_{\vec{a}}\right\rangle:=\lambda \csc \left\{\lambda\left(\vec{a}^{\prime} \cdot \vec{\partial}\right)\right\} \cdot \frac{1}{\lambda} \sin \{\lambda(\vec{a} \cdot \vec{\partial})\} \delta\left(\vec{a}^{\prime}-\vec{a}\right)=\delta\left(\vec{a}^{\prime}-\vec{a}\right) .
$$

$\underline{\text { Difference two-form } \Omega_{2}^{\mathrm{D}}}$

$$
\Omega_{2}^{\mathrm{D}}=\int d \vec{\alpha} \int d \vec{\beta} w_{\lambda}(\vec{x}: \vec{\alpha}, \vec{\beta}) \Delta^{\vec{\alpha}} \wedge \Delta^{\vec{\beta}}
$$


Here $\wedge$ is nothing but the ordinary wedge product. The forms of higher degree can be constructed by using the operation $\Delta$ repeatedly.

Exterior difference operator $\Delta$

$$
\Delta \Omega_{2}^{\mathrm{D}}=\int d \vec{\gamma} \int d \vec{\alpha} \int d \vec{\beta} \nabla_{\vec{\gamma}} w_{\lambda}(\vec{x}: \vec{\alpha}, \vec{\beta}) \Delta^{\vec{\gamma}} \wedge \Delta^{\vec{\alpha}} \wedge \Delta^{\vec{\beta}}
$$

Since $\left[\nabla_{\vec{\alpha}}, \nabla_{\vec{\beta}}\right]=0, \Delta$ has the desired property $\Delta \Delta=0$.

$\underline{\text { Interior product } i_{\nabla_{\vec{a}}}}$

$$
i_{\nabla_{\vec{\gamma}}}\left(\Delta^{\vec{\alpha}} \wedge \Delta^{\vec{\beta}}\right)=\delta(\vec{\gamma}-\vec{\alpha}) \Delta^{\vec{\beta}}-\delta(\vec{\gamma}-\vec{\beta}) \Delta^{\vec{\alpha}}
$$

Lie difference $\mathcal{L}_{X^{\mathrm{D}}}$

$$
\mathcal{L}_{X^{\mathrm{D}}}=\Delta \cdot i_{X^{\mathrm{D}}}+i_{X^{\mathrm{D}}} \cdot \Delta
$$

We obtain relations among operators $\Delta, i_{X^{\mathrm{D}}}$, and $\mathcal{L}_{X^{\mathrm{D}}}$ in the similar form to the continuous case. This means that the operators $\left(d, i_{X}, \mathcal{L}_{X}\right)$ in the differential geometry are replaced by $\left(\Delta, i_{X^{\mathrm{D}}}, \mathcal{L}_{X^{\mathrm{D}}}\right)$ here.

\subsection{Difference operator approach to the Moyal Quantization}

Now let us consider the case that $M$ is symplectic, i.e. the phase space. I only treat the two-dimensional physical phase space $\vec{x}=(p, x)$ for simplicity. The following discussion is easily generalized to the higher dimensional case.

In the classical mechanics, the Poisson bracket is geometrically realized as the action of the Hamiltonian vector field on a function such as $X_{f} g=-\{f, g\}$. Then it seems natural to think that an analogous formulation for the Moyal bracket should exist. I will show that the difference analogue of vector fields enables us to realize such concept. To this end I first propose a difference version of the Hamiltonian vector field $X_{f}$ as follows:

$$
X_{f}^{\mathrm{D}}=\int d \vec{a} v_{\lambda}[f](\vec{x}, \vec{a}) \nabla_{\vec{a}}
$$

where

$$
v_{\lambda}[f](\vec{x}, \vec{a})=\frac{1}{(2 \pi)^{2}} \int d \vec{b} e^{i(\vec{a} \times \vec{b})} f(\vec{x}+i \vec{b})
$$

In this expression $\vec{a} \times \vec{b}$ means the vector product of $\vec{a}$ and $\vec{b}$, and it inherits the symplectic structure from the phase space. If we apply $X_{f}^{\mathrm{D}}$ on a function $g$, we have the following desired expression:

$$
X_{f}^{\mathrm{D}} g=-\left.\frac{1}{\lambda} \sin \left[\lambda\left(\frac{\partial}{\partial x_{1}} \frac{\partial}{\partial p_{2}}-\frac{\partial}{\partial p_{1}} \frac{\partial}{\partial x_{2}}\right)\right] f\left(p_{1}, x_{1}\right) g\left(p_{2}, x_{2}\right)\right|_{p, x}=:-\{f, g\}_{\mathrm{M}}
$$


Therefore $X_{f}^{\mathrm{D}} g$ can be regarded as a difference operator representation of the Moyal bracket. In the $\lambda \rightarrow 0$ limit, (2.10) becomes $X_{f}$ and (2.12) the Poisson bracket. Moreover the relation as

$$
\left[X_{f}^{\mathrm{D}}, X_{g}^{\mathrm{D}}\right]=-X_{\{f, g\}_{\mathrm{M}}}^{\mathrm{D}}
$$

is verified by explicitly using (2.10). In this sense $X_{f}^{\mathrm{D}}$ is meaningful as the vector field on the phase space. This form of algebra has been studied in other context [22 and is sometimes called the Moyal bracket algebra (or the sine algebra).

Remark that the difference two-form corresponding to the symplectic form $\omega$ can be considered:

$$
\Omega:=\frac{1}{2 i \lambda} \int d a \int d b e^{-i \lambda(\vec{a} \times \vec{b})} \Delta^{\vec{a}} \wedge \Delta^{\vec{b}}
$$

For the two-form we can show

$$
i_{X_{f}^{\mathrm{D}}} \Omega=\Delta f .
$$

This relation is analogous to the definition of the Hamiltonian vector field as $i_{X_{f}} \omega=d f$. Therefore I interpret (2.14) as a difference version of the symplectic form.

Next I establish the way to reconstruct the Moyal quantization method by identifying the parameter $\lambda$ with Planck's constant as $\lambda=\hbar / 2$. There are several methods which connect an (quantum) observable on the Hilbert space with a function on the phase space. If we introduce the Moyal bracket as a quantum version of the Poisson bracket, we must fix the ordering of the operators along the so-called Weyl correspondence [1]. On the basis of the procedure of the phase space quantization, I regard $X_{A}^{\mathrm{D}}$ as the object corresponding to the phase space function $A$. This means that $X_{A}^{\mathrm{D}}$ takes the place of the observable in our description.

In addition to this, an expectation value of $X_{A}^{\mathrm{D}}$ must be introduced and it should be equivalent to the expectation value in the ordinary quantum mechanics. For this purpose I define the one-form associated with the Wigner distribution $F_{\mathrm{w}}$ [2] (see Appendix A) by

$$
\Omega_{F_{\mathrm{w}}}=\int d \vec{a} \int d \vec{b} e^{-i(\vec{a} \times \vec{b})} F_{\mathrm{w}}(\vec{x}+i \vec{b}) \Delta^{\vec{a}}
$$

Using the one-form and the orthogonality between $\nabla_{\vec{a}}$ and $\Delta^{\vec{a}}$, we actually obtain

$$
\left\langle\Omega_{F_{\mathrm{w}}}, X_{A}^{\mathrm{D}}\right\rangle=\int d \vec{x} F_{\mathrm{w}}(\vec{x}) A(\vec{x})
$$

The right-hand side is nothing but the expectation value of $A$ in the phase space quantization. Hence we can say that the left-hand side is a new description of the expectation value. (See 21] for more detail.)

We can also consider the time evolution of (2.17). The time dependence of $\left\langle\Omega_{F_{\mathrm{w}}}, X_{A}^{\mathrm{D}}\right\rangle$ can be two fold: in the Heisenberg picture we have $\left\langle\Omega_{F_{\mathrm{w}}}, X_{A}^{\mathrm{D}}\right\rangle_{t}=\left\langle\Omega_{F_{\mathrm{w}}}, X_{A}^{\mathrm{D}}(t)\right\rangle$, while in the Schrödinger 
picture we have $\left\langle\Omega_{F_{\mathrm{w}}}(t), X_{A}^{\mathrm{D}}\right\rangle$, which must be equivalent. (In the following discussion, we assume that $F_{\mathrm{w}}$ (in Heisenberg) and $A$ (in Schrödinger) have no explicit time dependence.) If we take the Heisenberg picture, the time evolution of a physical observable (A.9) of Appendix A should follow to one of $X_{A}^{\mathrm{D}}$. In fact, if the static Hamiltonian $H$ is given, the equation

$$
\frac{d}{d t} X_{A}^{\mathrm{D}}=-\left[X_{A}^{\mathrm{D}}, X_{H}^{\mathrm{D}}\right]
$$

enables us to make such an interpretation. Since $X_{A}^{\mathrm{D}}(t)$ is written as $X_{A(t)}^{\mathrm{D}}$, this equation is

physically equivalent to (A.9) in Appendix A, and $A(t)=e^{X_{H}^{\mathrm{D}} t} A$. Then from (2.13), we obtain

$$
\frac{d}{d t}\left\langle\Omega_{F_{\mathrm{w}}}, X_{A}^{\mathrm{D}}(t)\right\rangle=\left\langle\Omega_{F_{\mathrm{w}}}, X_{\{A(t), H\}_{\mathrm{M}}}^{\mathrm{D}}\right\rangle .
$$

The right-hand side of (2.19) is identical with $\left\langle\Omega_{\left\{H, F_{\mathrm{w}}(t)\right\}_{\mathrm{M}}}, X_{A}^{\mathrm{D}}\right\rangle$ (see Appendix B). Hence we obtain an equation which $\Omega_{F_{\mathrm{w}}}(t)=\Omega_{F_{\mathrm{w}}(t)}$ must satisfy in the Schrödinger picture:

$$
\frac{d}{d t} \Omega_{F_{\mathrm{w}}}(t)=\Omega_{\left\{H, F_{\mathrm{w}}(t)\right\}_{\mathrm{M}}}
$$

Here we used $F_{\mathrm{w}}(t):=e^{-X_{H}^{\mathrm{D}} t} F_{\mathrm{w}}$. It is clear that this equation corresponds to (A.10).

All these are our reconstruction of the Moyal quantization. Though these results have been known by using other methods, the difference operator approach must provide a new insight into the Moyal quantization not only formally but also practically.

\section{Application to the KP-hierarchy}

In the difference analogue of the vector field approach, we notice that it has property different from the ordinary vector field, i.e. infinite dimensionality. Even if we start from the finite-dimensional phase space, its quantum version leads us to an infinite-dimensional vector field in a sense that the vector $\vec{a}$ plays the role of the indices. Since $\left\{X^{\mathrm{D}}\right\}$ constitutes an infinitedimensional Lie algebra, it may have some connection with symmetry of nonlinear integrable systems. The purpose of this section is to clarify it by applying our methods to the theory of the KP-hierarchy.

First we consider the complex-valued phase space $(z, \zeta)$ instead of $(p, x)$ and a function on it is assumed to be expanded in the formal Laurent series as

$$
A(z, \zeta)=\sum_{m \in \mathbf{Z}} \sum_{n \in \mathbf{Z}} a_{m n} z^{m} \zeta^{n}
$$

In this case $X_{A}^{\mathrm{D}}$ becomes

$$
X_{A}^{\mathrm{D}}=\frac{1}{\lambda} \sum_{m, n} a_{m n} z^{m} \zeta^{n} \sin \left\{\lambda\left(n \partial_{\ln z}-m \partial_{\ln \zeta}\right)\right\}=: \sum_{m, n} a_{m n} z^{m} \zeta^{n} \nabla_{m n}
$$


The factor $\nabla_{m n}$ plays a role of $\nabla_{\vec{a}}$ in (2.1). More precisely this expression is equivalent to the form that the integration is carried out in (2.1). If we define the "components" $X_{m n}^{\mathrm{D}}$ of $X_{A}^{\mathrm{D}}$ by $z^{m} \zeta^{n} \nabla_{m n}$, it constitutes a basis of the following Lie algebra:

$$
\left[X_{m_{1} n_{1}}^{\mathrm{D}}, X_{m_{2} n_{2}}^{\mathrm{D}}\right]=\frac{1}{\lambda} \sin \left\{\lambda\left(n_{1} m_{2}-n_{2} m_{1}\right)\right\} X_{m_{1}+m_{2}, n_{1}+n_{2}}^{\mathrm{D}}
$$

22, and also that $X_{m n}^{\mathrm{D}}$ satisfy $(2.13)$ ).

Next I define the corresponding object of the Wigner distribution. In the theory of the KPhierarchy, the Baker-Akhiezer function plays the role of the wave function of quantum physics in the sense that it satisfies the linear equations of the inverse problem associated with the nonlinear differential equations. Then it is preferable to compose the "distribution" from the Baker-Akhiezer function by regarding the spectral parameter as the coordinate of the phase space $(z, \zeta)$. But we must remember that the KP-hierarchy is the system which has multi-time evolution; and such evolution is generated by the operation of the pseudo-differential operator. Moreover the complex conjugate of the wave function in the quantum mechanics is turned into the formal adjoint in this case. Taking these facts into consideration, the following functional is suitable for our purpose:

$$
F_{\mathrm{KP}}(z, \zeta):=\int d x \sum_{l \in \mathbf{Z}} w\left(q^{\frac{l}{2}} z\right) w^{*}\left(q^{-\frac{l}{2}} z\right) \zeta^{-l}
$$

where the parameter $\lambda$ is fixed to $-i \ln q(|q|<1)$, and $w\left(w^{*}\right)$ stands for the (adjoint) BakerAkhiezer function. The integration over the variable $y$ in (A.3) of Appendix A corresponds to the summation over $l$. The integration over $x$ does not exist in (A.3). This is because the Baker-Akhiezer function has $x$-dependence (see (C.3) in Appendix C), while in the quantum wave function there is no such dependence. (Remark that the variable $x$ in the Baker-Akhiezer function does not mean position since in this case $z$ and $\zeta$ are the coordinates of the phase space.) Hence I smear out the $x$-dependence of the Baker-Akhiezer function by taking an "average" in the definition of $F_{\mathrm{KP}}(z, \zeta)$.

We can define the one-form $\Omega_{F_{\mathrm{KP}}}$ associated with (3.3) in the same way as discussed in the previous section. It is clear that (3.3) is formally expanded as $F_{\mathrm{KP}}=\sum_{m, n} f_{m n} z^{m} \zeta^{n}$. Then first we introduce the dual basis $\Delta^{m n}$ of $\nabla_{m n}$ and define $\Omega_{F_{\mathrm{KP}}}$ by

$$
\Omega_{F_{\mathrm{KP}}}(z, \zeta)=\sum_{m, n} f_{m n} z^{m} \zeta^{n} \Delta^{m n}
$$

${ }^{*}$ The Moyal bracket is now expressed as

$$
\{f, g\}_{\mathrm{M}}=-\left.\frac{1}{\lambda} \sin \left\{\lambda\left(\frac{\partial}{\partial \ln z_{1}} \frac{\partial}{\partial \ln \zeta_{2}}-\frac{\partial}{\partial \ln \zeta_{1}} \frac{\partial}{\partial \ln z_{2}}\right)\right\} f\left(z_{1}, \zeta_{1}\right) g\left(z_{2}, \zeta_{2}\right)\right|_{z, \zeta} .
$$


The "expectation value" now becomes

$$
\left\langle\Omega_{F_{\mathrm{KP}}}, X_{A}^{\mathrm{D}}\right\rangle:=-\oint \frac{d z}{2 \pi i z} \oint \frac{d \zeta}{2 \pi i \zeta} F_{\mathrm{KP}}(z, \zeta) A(z, \zeta)=\sum_{m, n} f_{m n} a_{m n}
$$

on the basis of the orthogonality relation as $\left\langle\Delta^{m n}, \nabla_{m^{\prime} n^{\prime}}\right\rangle=\delta_{m m^{\prime}} \delta_{n n^{\prime}}$. Here each integral means to pick up the coefficient of $z^{-1}\left(\zeta^{-1}\right.$, resp) term. In particular if we use $X_{m n}^{\mathrm{D}}$, we obtain

$$
f_{m n}=\left\langle\Omega_{F_{\mathrm{KP}}}, X_{m n}^{\mathrm{D}}\right\rangle=-\int d x \oint \frac{d z}{2 \pi i z} z^{m} w\left(q^{\frac{n}{2}} z\right) w^{*}\left(q^{-\frac{n}{2}} z\right)
$$

It is interesting to consider this result in connection with the additional flow $\partial_{k l}$ discussed in [17, 18]. The use of $\partial_{k l}$ enables us to represent the right-hand side of (3.6) in a simpler form as

$$
\int d x \mathcal{D}_{m n}\left(-\frac{\partial}{\partial x} \ln \tau\right)
$$

See Appendix $\mathrm{C}$ for more detail. In this expression, $\tau$ is the tau function of the KP-hierarchy and $\mathcal{D}_{m n}$ is defined as

$$
\mathcal{D}_{m n}:=q^{\frac{n m}{2}} \sum_{j, l=0}^{\infty} c_{j l} \frac{(n \lambda)^{j}}{j !} \partial_{m+l-1, l}
$$

where $c_{j l}$ is

$$
c_{j l}=\sum_{\alpha=1}^{l} \frac{(-1)^{l-\alpha} \alpha^{j}}{(l-\alpha) ! \alpha !}(j, l \geq 1), \quad c_{l 0}=c_{0 l}=\delta_{l, 0} .
$$

The quantity $-\partial_{x} \ln \tau$ is nothing but the solution of the KP-hierarchy. This means that $\Omega_{F_{\mathrm{KP}}}$ induces the $\mathrm{KP}$ solution. On the other hand, $\mathcal{D}_{m n}$ comes from $X_{m n}^{\mathrm{D}}$. In this sense the additional symmetry has been given its alternative interpretation from the discretization point of view. Strictly speaking, the subscrips $m$ and $n$ run over the whole integer, while one of the subscripts of the additional flow $\partial_{k l}$ the positive integers (see Appendix $\mathrm{C}$ ). Then the correspondence between $\mathcal{D}_{m n}$ and $\partial_{k l}$ is not one-to-one. Nevertheless the essential point is that $\mathcal{D}_{m n}$ can be represented by using the additional flow. If we want to let the correspondence be one-to-one, we must truncate the area over which one of the subscripts of $\mathcal{D}_{m n}$ runs. Such a situation can be realized, for instance, by imposing some condition on $a_{m n}$ and/or $f_{m n}$. The additional symmetry is known as a kind of the so-called $W$-symmetry [23]. Therefore above consideration leads us to think that the discretization which preserves integrability is closely related to the $W$-symmetry in general since such symmetry is believed to possess the universal properties of various integrable systems.

If we define $\mathcal{D}_{A}:=\sum_{m, n} a_{m n} \mathcal{D}_{m n}$, we at last obtain the "expectation value" as the following functional:

$$
\left\langle\Omega_{F_{\mathrm{KP}}}, X_{A}^{\mathrm{D}}\right\rangle=\int d x \mathcal{D}_{A}\left(-\frac{\partial}{\partial x} \ln \tau\right)=: \tilde{A}(t)
$$


Let us investigate the meaning of the functional $\tilde{A}(t)$. The (multi-) time evolution of $\tilde{A}(t)$ is obtained by taking differential with respect to $t_{r}$ :

$$
\frac{\partial}{\partial t_{r}} \tilde{A}=-\int d x \mathcal{D}_{A}\left(\frac{\partial}{\partial t_{r}} \frac{\partial}{\partial x} \ln \tau\right)=:-\int d x \mathcal{D}_{A} J_{r}
$$

Here $J_{r}=\partial_{r} \partial_{x} \ln \tau$ is the first integral of the KP-hierarchy, i.e. $H_{r}=\int d x J_{r}$ is the Hamiltonian of the KP-hierarchy [19]. Since the non-commutative flow $\mathcal{D}_{A}$ generates the independent symmetry from the ordinary KP-flow $\partial_{r}$, this equation means that the time evolution of the "expectation value" naturally yields the Hamiltonian structure of the KP-hierarchy. For example, if we take the derivative with respect to another time variable $t_{r^{\prime}}$, we obtain

$$
\frac{\partial}{\partial t_{r^{\prime}}} \int d x \mathcal{D}_{A} J_{r}=0
$$

In this sense, the right-hand side of (3.10) is nothing but the Hamiltonian of the KP-hierarchy induced by the non-commutative flow. This fact is not surprising: The form such as $w \cdot w^{*}$ is known as the residue (with respect to the pseudo-differential operator) of a "resolvent" of the Hamiltonian mapping in the KP-hierarchy [19]. And turning back to our definition of $F_{\mathrm{KP}}$, we find that $F_{\mathrm{KP}}$ essentially consists of such bilinear form although it is constructed by analogy with the Wigner distribution. Hence the emergence of the Hamiltonian is natural.

On the other hand, we can introduce the vector field associated with the functional $\tilde{A}$. Using it, the right-hand side of (3.10) is formally defined as

$$
-\int d x \mathcal{D}_{A} J_{r}=: \mathcal{D}_{\tilde{A}} \cdot H_{r}
$$

The action of $\mathcal{D}_{\tilde{A}}$ on the functional $H_{r}$ has the same structure as (2.12). Therefore we can interpret that $\mathcal{D}_{\tilde{A}} \cdot H_{r}$ provides the Moyal bracket structure in the KP theory, i.e. we can write down (3.10) as follows:

$$
\frac{\partial}{\partial t_{r}} \tilde{A}=\left\{\tilde{A}, H_{r}\right\}_{\mathrm{M}}^{(\mathrm{KP})}
$$

I have shown that the difference operator approach to the Moyal quantization can be applied to the KP theory. It is remarkable that the integrability of the KP-hierarchy ( $\tau$ function, Hamiltonian structure) is naturally led from the particular forms of $F_{\mathrm{KP}}$ and $\tilde{A}$. On the other hand the algebraic structure of $X_{f}^{\mathrm{D}}$ discussed in Section 2 restricts the form of $\Omega_{F_{\mathrm{KP}}}$. From these facts I like to emphasize that the Moyal bracket structure implies the origin of the integrability of the system. 


\section{Deformation of the Poisson structure of the soliton system}

In the preceding section I revealed the Moyal bracket structure in the KP system by considering the time evolution of the functional $\tilde{A}$. Then I arrive at a speculation that in integrable systems such structure can be constructed manifestly. I will examine in this section whether it is appropriate.

First I notice that from (3.8) we can associate $X_{m n}^{\mathrm{D}}$ with the holomorphic vector field. For the holomorphic function $f(z)=\sum f_{m} z^{m+1}$, the operator which generates the infinitesimal conformal transformation is defined as

$$
L_{f}=f(z) \partial_{z}=\sum_{m \in \mathbf{Z}} f_{m} z^{m+1} \partial_{z}=: \sum_{m \in \mathbf{Z}} f_{m} L_{m}
$$

This can be understood as the vector field in the following sense:

$$
\left[L_{f}, L_{g}\right]=L_{L_{f} g-L_{g} f}
$$

The component $L_{m}$ is the basis of the Virasoro algebra. By analogy with the case of the Hamiltonian vector field, I provide the difference analogue of (4.1) by

$$
L_{f}^{\mathrm{D}}:=\sum_{m \in \mathbf{Z}} \sum_{n \in \mathbf{Z}_{\geq 0}} q^{-\frac{n(m+2)}{2}} f_{m} z^{m} q^{n\left(z \partial_{z}+\frac{m}{2}\right)}=: \sum_{m, n} q^{-\frac{n(m+2)}{2}} f_{m} L_{m n}^{\mathrm{D}}
$$

Remark that the subscript $n$ is truncated to the non-negative integer (see the discussion above). In this case $L_{f}^{\mathrm{D}}$ also constitutes a Lie algebra:

$$
\left[L_{f}^{\mathrm{D}}, L_{g}^{\mathrm{D}}\right]=L_{L_{f}^{\mathrm{D}} g-L_{g}^{\mathrm{D}} f}
$$

When the coefficient of the function $A(z, \zeta)=\sum a_{m n} z^{m} \zeta^{n}$ is given as $a_{m n}=q^{-n(m+2) / 2} a_{m}$, the "expectation value" of $A$ is written as follows:

$$
\left\langle\Omega_{F_{\mathrm{KP}}}, X_{A}^{\mathrm{D}}\right\rangle=\int d x\left[\oint \frac{d z}{2 \pi i z}\left(L_{A}^{\mathrm{D}} V(z)\right) V^{*}(z)\right]\left(-\frac{\partial}{\partial x} \ln \tau\right) .
$$

From this fact, (4.4) is recognized as a discretization of the holomorphic vector field which corresponds to the Moyal deformation. Schematically, the correspondence is illustrated as

$$
\begin{aligned}
& X_{f} g \text { (Poisson) } \longleftrightarrow L_{f} g-L_{g} f \\
& X_{f}^{\mathrm{D}} g \text { (Moyal) } \longleftrightarrow L_{f}^{\mathrm{D}} g-L_{g}^{\mathrm{D}} f
\end{aligned}
$$

In [15] we showed that such type of $q$-difference operator realization of the $w_{1+\infty}$ algebra can be understood as a specific realization of the discretization of the conformal symmetry. (Actually 
it was constructed by using $L_{m n}^{\mathrm{D}}$ in (4.3).) Also in this sense above interpretation is naturally achieved. The appearance of the holomorphic vector fields also implies that my procedure of the expectation value has mathematically similar background to the polarization process in the geometric quantization [24]. Then the relation between the Moyal quantization and the geometric quantization might be clarified from our point of view.

The above diagram for the holomorphic vector fields indicates the existence of some new bracket corresponding to the Moyal bracket. In the following discussion, I will show such evidence by considering the $\mathrm{KdV}$ equation $u_{t}-6 u u_{x}+u_{x x x}=0$ as a simple example. For the solution $u(x, t)$, let us define the functional $\mathcal{L}_{m}$ as

$$
\mathcal{L}_{m}=\int d x x^{m+1} u
$$

It is known [25] that the following bracket constitutes the Virasoro algebra:

$$
\begin{aligned}
\left\{\mathcal{L}_{m}, \mathcal{L}_{n}\right\} & =\int d x x^{m+1}(u \partial+\partial u) x^{n+1} \quad(\partial=\partial / \partial x) \\
& =(m-n) \mathcal{L}_{m+n}
\end{aligned}
$$

This bracket has the properties of the Poisson bracket and provides a part of the second Hamiltonian structure of the KdV equation?. The fact can be contrasted to the case of the holomorphic vector field. Now I propose a functional corresponding to the difference analogue of the vector field $L_{m n}^{\mathrm{D}}$ in (4.1) as

$$
\mathcal{L}_{m n}^{\mathrm{D}}=\int d x q^{-n} x^{m} u
$$

under the definition of the new bracket:

$$
\left\{\mathcal{L}_{m n}^{\mathrm{D}}, \mathcal{L}_{m^{\prime} n^{\prime}}^{\mathrm{D}}\right\}_{q}:=\int d x q^{-n} x^{m}\left(q^{n^{\prime} x \partial} u q^{n x \partial}-q^{-n^{\prime} x \partial} u q^{-n x \partial}\right) q^{-n^{\prime}} x^{m^{\prime}}
$$

If we take the partial integration

$$
\int d x f(x)\left(q^{x \partial} g(x)\right)=\int d x\left(q^{-x \partial} f(x)\right) g(x),
$$

the right-hand side of (4.9) yields

$$
\left(q^{n m^{\prime}-n^{\prime} m}-q^{-n m^{\prime}+n^{\prime} m}\right) \mathcal{L}_{m+m^{\prime} n+n^{\prime}}^{\mathrm{D}} .
$$

After substitution of $-i \ln q$ for $\lambda$ and some change of normalization, (4.9) becomes the same algebra as (3.2). In this sense the term

$$
q^{n^{\prime} x \partial} u q^{n x \partial}-q^{-n^{\prime} x \partial} u q^{-n x \partial}
$$

${ }^{*}$ More precisely, the term as $\partial^{3}$ must be added to $(u \partial+\partial u)$ in $(4.7)$ and this term is nothing but the origin of the center. But in our naive discussion here, we temporary ignore it. 
can be interpreted as a deformation (discretization) of the term as $u \partial+\partial u$. More rigorous treatment may lead us to the Moyal-like deformation of the Hamiltonian structure of the KdV equation. Furthermore it is interesting to generalize the above investigation to the integrable hierarchy. Recently the $q$-deformation of the integrable hierarchy was discussed in [26]. There the $q$-difference version of the pseudo-differential operator plays an essential role. Such a generalization may have deep connection with it.

\section{Summary and Discussion}

First I summarize the results of the manuscript.

In section 2 , the difference analogue of the vector fields $X^{\mathrm{D}}$ and its dual form $\Omega$ were defined; and their geometrical properties were discussed. In the reformulation of the quantum mechanics, I proposed the difference Hamiltonian vector field $X_{A}^{\mathrm{D}}$ and its dual form $\Omega_{F_{\mathrm{w}}}$ which was associated with the Wigner distribution. The expectation value of an observable could be constructed by pairing of $X_{A}^{\mathrm{D}}$ with $\Omega_{F_{\mathrm{w}}}$. The time evolution of observable and the Wigner distribution was rewritten in our prescription. All these results were already known in the context of the phase space quantization. Although I paraphrased them again by using the difference operator and reformulated them since such construction had the possibility to provide new insight into the Moyal quantization.

In section 3, I showed that the preceding formulation was applicable to the theory of the KP-hierarchy: The "distribution" $F_{\mathrm{KP}}$ was defined by using the Baker-Akhiezer function in this case. The one-form $\Omega_{F_{\mathrm{KP}}}$ is connected with the KP solution; and the deformed vector field $X_{A}^{\mathrm{D}}$ induces the non-commutative (or $w_{1+\infty}$ ) flow of the KP solutions. The time evolution of the functional $\tilde{A}$ which corresponds to the "expectation value" yields the Hamiltonian structure of the KP-hierarchy. After all it has been clarified that the integrability of the KP-hierarchy followed to the nature of $\Omega_{F_{\mathrm{KP}}}$ and of the Moyal bracket.

In addition to these formal study of the Moyal quantization, in Section 4, I discussed $X_{A}^{\mathrm{D}}$ was related to the algebraic structure which was characteristic of integrable hierarchies: The component $X_{m n}^{\mathrm{D}}$ is identical with a realization of the difference analogue of the conformal algebra. Moreover this fact led us to the Moyal-like deformation of the Hamiltonian structure of the $\mathrm{KdV}$ equation such as $\left\{\mathcal{L}_{m n}^{\mathrm{D}}, \mathcal{L}_{m^{\prime} n^{\prime}}^{\mathrm{D}}\right\}_{q}$. Therefore the difference analogues of the integrable system provided by the Moyal structure should preserve their integrability.

The Moyal bracket structure in the integrable system was also discussed in other context [27]. There the variables $x$ and $z$ are regarded as the canonical variable. Originally, such description was made in the theory of the dispersionless KP-hierarchy 28. In this case, the pseudodifferential operator plays a role of the quantum operator, i.e. the following correspondence is 
realized:

$$
P\left(x, \partial_{x}\right)=\sum_{j} p_{j}(x) \partial_{x}^{j} \longmapsto \mathcal{P}(x, z)=\sum_{j} p_{j}(x) z^{j} .
$$

This correspondence is equivalent to take other type of operator-ordering, so-called the standard ordering [29]-[31]. Nevertheless I think that the difference operator approach discussed above is still effective here. I will report it elsewhere.

\section{Acknowledgements}

I would like to thank Satoru Saito for many valuable discussions and careful reading of this manuscript.

\section{Appendix A.}

In this appendix I briefly review the phase space quantization.

Let us consider the one-dimensional system in the state $|\phi\rangle$. (It is not difficult to generalize to higher dimensional case.) First we introduce the characteristic operator:

$$
\hat{M}(\hat{p}, \hat{x} ; \tau, \theta)=e^{i(\tau \hat{p}+\theta \hat{x})},
$$

where $\tau$ and $\theta$ have the same dimension as one of position and momentum, respectively. The expectation value $M$ of $\hat{M}$ in state $|\phi\rangle$ can be written as

$$
M(\tau, \theta)=\int d x \phi\left(x+\frac{\hbar}{2} \tau\right) \phi^{*}\left(x-\frac{\hbar}{2} \tau\right) e^{i \theta x} .
$$

We define the Wigner distribution as the Fourier transformation of the above equation:

$$
F_{\mathrm{w}}(p, x)=\frac{1}{(2 \pi)^{2}} \int d \tau \int d \theta M(\tau, \theta) e^{-i(\tau p+\theta x)}=\frac{1}{2 \pi} \int d y \phi\left(x+\frac{\hbar}{2} y\right) \phi^{*}\left(x-\frac{\hbar}{2} y\right) e^{-i p y} .
$$

The Wigner function corresponds to the classical probability density on the phase space and reduces to it in the limit of $\hbar \rightarrow 0$, but in general it might take negative values and hence does not have the meaning of a probability density except in the classical limit.

Now let us consider an arbitrary operator of $\hat{p}, \hat{x}$, say $\hat{A}(\hat{p}, \hat{x})$ as follows:

$$
\hat{A}(\hat{p}, \hat{x})=\int d \tau \int d \theta a(\tau, \theta) e^{i(\tau \hat{p}+\theta \hat{x})} .
$$

In this expression the operator is taken an appropriate representation, e.g. $\hat{p}=-i \hbar \partial / \partial \hat{x}$ in the position representation. We assume the corresponding phase space function has the following form as

$$
A(p, x)=\int d \tau \int d \theta a(\tau, \theta) e^{i(\tau p+\theta x)}
$$


This correspondence is called the Weyl correspondence. Remark that such correspondence fixes the operator ordering (Weyl ordering). For example, the classical quantity $p^{m} x^{n}$ becomes

$$
\frac{1}{2^{n}} \sum_{r=0}^{n}\left(\begin{array}{c}
n \\
r
\end{array}\right) \hat{x}^{n-r} \hat{p}^{m} \hat{x}^{r}
$$

If we take the expectation value of $\hat{A}$ in the state $|\phi\rangle$, we get

$$
\langle\phi|\hat{A}| \phi\rangle=\int d p \int d x F_{\mathrm{w}}(p, x) A(p, x)
$$

The expectation value of the commutator $[\hat{A}, \hat{B}]$ becomes

$$
\langle\phi|[\hat{A}(\hat{p}, \hat{x}), \hat{B}(\hat{p}, \hat{x})]| \phi\rangle=i \hbar \int d p \int d x F_{\mathrm{w}}(p, x)\{A, B\}_{\mathrm{M}}
$$

The Moyal bracket reduces to the Poisson bracket in the $\hbar \rightarrow 0$ limit. In this formalism, the time evolution of the observable is written as

$$
\frac{d}{d t} A(t)=\{A(t), H\}_{\mathrm{M}}
$$

and it corresponds to the Heisenberg picture. We also consider the Schrödinger picture in which the Wigner distribution depends on time. It originates from the density matrix $\hat{\rho}$, and in the statistical treatment the expectation value is denoted by $\operatorname{Tr}(\hat{\rho} \hat{A})$. In this sense we can consider the time evolution of the Wigner distribution and it becomes

$$
\frac{d}{d t} F_{\mathrm{w}}(t)=\left\{H, F_{\mathrm{w}}(t)\right\}_{\mathrm{M}}
$$

For detailed treatments of the dynamics of these equations, see [2]-[10].

\section{Appendix B.}

In this appendix I show that the relation $\left\langle\Omega_{F_{\mathrm{w}}}, X_{\{A(t), H\}_{\mathrm{M}}}^{\mathrm{D}}\right\rangle=\left\langle\Omega_{\left\{H, F_{\mathrm{w}}(t)\right\}_{\mathrm{M}}}, X_{A}^{\mathrm{D}}\right\rangle$ holds. To verify the relation I first prove

$$
\int d \vec{x} F_{\mathrm{w}}\left(X_{H}^{\mathrm{D}} A\right)=-\int d \vec{x}\left(X_{H}^{\mathrm{D}} F_{\mathrm{w}}\right) A
$$

Proof of $B .1)$ :

(l.h.s. of $(\mathrm{B} .1))=-\int d \vec{x} F_{\mathrm{w}}(\vec{x})\{A, H\}_{\mathrm{M}}(\vec{x})$ 


$$
\begin{aligned}
& =-\frac{1}{(2 \pi)^{2}} \int d \vec{x} F_{\mathrm{w}}(\vec{x}) \int d \vec{a} \int d \vec{b} \sin (\vec{a} \times \vec{b}) A(\vec{x}+i \vec{b}) H(\vec{x}+i \vec{a}) \\
& =-\frac{1}{(2 \pi)^{2}} \int d \vec{x} \int d \vec{a} \int d \vec{b} \sin (\vec{a} \times \vec{b}) F_{\mathrm{w}}(\vec{x}-i \vec{b}) H(\vec{x}+i \vec{a}-i \vec{b}) A(\vec{x}) \\
& =\frac{1}{(2 \pi)^{2}} \int d \vec{x} \int d \vec{a} \int d \vec{b} \sin (\vec{a} \times \vec{b}) F_{\mathrm{w}}(\vec{x}+i \vec{b}) H(\vec{x}+i \vec{a}) A(\vec{x}) \\
& =\int d \vec{x}\left\{H, F_{\mathrm{w}}\right\}_{\mathrm{M}}(\vec{x}) A(\vec{x})=(\text { r.h.s. of }(\mathrm{B} .1))
\end{aligned}
$$

Combining (B.1) with the relations as

$$
A(t)=e^{i X_{H}^{\mathrm{D}} t} A, \quad F_{\mathrm{w}}(t)=e^{-i X_{H}^{\mathrm{D}} t} F_{\mathrm{w}},
$$

we can make sure the following equality:

$$
\left\langle\Omega_{F_{\mathrm{w}}}, X_{\left\{\exp \left(i X_{H}^{\mathrm{D}} t\right) A, H\right\}_{\mathrm{M}}}^{\mathrm{D}}\right\rangle=\left\langle\Omega_{\left\{H, \exp \left(-i X_{H}^{\mathrm{D}} t\right) F_{\mathrm{w}}\right\}_{\mathrm{M}}}, X_{A}^{\mathrm{D}}\right\rangle
$$

Proof of $(B .2)$ :

$$
\begin{aligned}
\text { (l.h.s. of (B.2)) } & =\int d \vec{x} F_{\mathrm{w}}\left\{e^{i X_{H}^{\mathrm{D}} t} A, H\right\}_{\mathrm{M}}=\sum_{n=0}^{\infty} \frac{(i t)^{n}}{n !} \int d \vec{x} F_{\mathrm{w}}\left\{\left(X_{H}^{\mathrm{D}}\right)^{n} A, H\right\}_{\mathrm{M}} \\
& =\sum_{n=0}^{\infty} \frac{(i t)^{n}}{n !} \int d \vec{x} F_{\mathrm{w}}\left(\left(X_{H}^{\mathrm{D}}\right)^{n+1} A\right) \\
& =-\sum_{n=0}^{\infty} \frac{(i t)^{n}}{n !} \int d \vec{x}\left(X_{H}^{\mathrm{D}} F_{\mathrm{w}}\right)\left(\left(X_{H}^{\mathrm{D}}\right)^{n} A\right) \\
& =\sum_{n=0}^{\infty} \frac{(i t)^{n}}{n !} \int d \vec{x}\left(\left(X_{H}^{\mathrm{D}}\right)^{2} F_{\mathrm{w}}\right)\left(\left(X_{H}^{\mathrm{D}}\right)^{n-1} A\right) \\
& =\cdots \\
& =(-1)^{n+1} \sum_{n=0}^{\infty} \frac{(i t)^{n}}{n !} \int d \vec{x}\left(\left(X_{H}^{\mathrm{D}}\right)^{n+1} F_{\mathrm{w}}\right) A \\
& =\int d \vec{x}\left\{H, F_{\mathrm{w}}(t)\right\}_{\mathrm{M}} A=(\text { r.h.s. of }(\mathrm{B} .2))
\end{aligned}
$$




\section{Appendix C.}

In this appendix I explain the non-commutative flow of the KP-hierarchy and derive (3.7).

In contrast to the KP flow $\partial_{r}=\partial / \partial t_{r}$, we can consider the flow $\partial_{k l}$ [17, 18] which do not commute with each other but commute with $\partial_{r}$. In this sense this additional flow is nothing but the symmetry of the KP-hierarchy. First remember that the KP-hierarchy is the following linear system of equations:

$$
L w=z w, \quad \partial_{r} w=L_{+}^{r} w,
$$

where the Lax operator $L$ and the Baker-Akhiezer function $w$ are respectively given by

$$
\begin{aligned}
& L(x, t)=\partial+\sum_{j=1}^{\infty} u_{j}(x, t) \partial^{-j}, \quad(\partial=\partial / \partial x) \\
& w(z, t)=\left(1+\sum_{j=1}^{\infty} w_{j} \partial^{-j}\right) \exp \sum_{r} t_{r} z^{r}=: W e^{\xi},
\end{aligned}
$$

and ()$_{+}$denotes the purely differential operator part. We also describe $L$ by using the dressing operator $W$ as $W \partial W$. The first equation of (C.1) means that the operation of $L$ on the BakerAkhiezer function is equivalent to the production of $z$ to $w$. Similarly, the derivation $\partial_{z}$ to $w$ is written in terms of the pseudo-differential operators as

$$
\frac{\partial w}{\partial z}=W\left(\sum_{r=1}^{\infty} r t_{r} \partial^{r-1}\right) W^{-1} W e^{\xi}=: M w
$$

Then we get $z^{k} \partial_{z}^{l} w=M^{l} L^{k} w\left(k \in \mathbf{Z}, l \in \mathbf{Z}_{\geq 0}\right)$. It enables us to consider the vector field as

$$
\partial_{k l} w=-\left(M^{l} L^{k}\right)_{-} w
$$

Moreover between $z^{k} \partial_{z}^{l}$ and $\partial_{k l}$, there is a Lie algebra isomorphism $z^{k} \partial_{z}^{l} \mapsto \partial_{k l}$. Therefore $\partial_{k l}$ yields the relation as

$$
\left[\partial_{k l}, \partial_{k^{\prime} l^{\prime}}\right]=\sum_{j=1}^{\infty}\left\{\left(\begin{array}{c}
k \\
j
\end{array}\right)\left(\begin{array}{c}
l \\
j
\end{array}\right)-\left(\begin{array}{c}
k^{\prime} \\
j
\end{array}\right)\left(\begin{array}{c}
l^{\prime} \\
j
\end{array}\right)\right\} j ! \partial_{k+k^{\prime}-j, l+l^{\prime}-j}
$$

In general the operators spanned by the differential operators $\left\{z^{k} \partial_{z}^{l} ; k \in \mathbf{Z}, l \in \mathbf{Z}_{\geq 0}\right\}$ form an infinite-dimensional Lie algebra, and it is called $w_{1+\infty}$ [1]6].

Since $w\left(w^{*}\right)$ is rewritten by use of the vertex operator $V\left(V^{*}\right)$ and the $\tau$ function as

$$
\begin{aligned}
& w(z, t)=\frac{V(z) \tau}{\tau}=\frac{\tau(t-1 /[z])}{\tau(t)} e^{\xi}, \quad w^{*}(z, t)=\frac{V^{*}(z) \tau}{\tau}=\frac{\tau(t+1 /[z])}{\tau(t)} e^{-\xi} \\
& \left(t \pm 1 /[z]:=t_{1} \pm 1 / z, t_{2} \pm 1 / 2 z^{2}, \cdots\right)
\end{aligned}
$$


we obtain the action of $\partial_{k l}$ on the $\tau$ function as

$$
\partial_{m+l, l} \partial \ln \tau=\oint \frac{d z}{2 \pi i}\left(z^{m+l} \partial_{z}^{l} w(z)\right) w^{*}(z)=\left[\oint \frac{d z}{2 \pi i}\left(z^{m+l} \partial_{z}^{l} V(z)\right) V^{*}(z)\right] \frac{\partial}{\partial x} \ln \tau
$$

15, 32, 33.

The derivation of (3.7) is shown as follows:

$$
\begin{aligned}
& \text { (r.h.s. of }(3.6))=-\int d x \oint \frac{d z}{2 \pi i z}\left(z^{m} q^{n\left(z \partial_{z}+\frac{m}{2}\right)} w(z)\right) w^{*}(z) \\
& =-q^{\frac{n m}{2}} \sum_{j, l=0}^{\infty} c_{j l} \frac{(n \lambda)^{j}}{j !} \int d x \oint \frac{d z}{2 \pi i z}\left(z^{m+l} \partial_{z}^{l} w(z)\right) w^{*}(z) \\
& =\int d x\left[q^{\frac{n m}{2}} \sum_{j, l=0}^{\infty} c_{j l} \frac{(n \lambda)^{j}}{j !} \partial_{m+l-1, l}\right]\left(-\frac{\partial}{\partial x} \ln \tau\right) \\
& =\int d x \mathcal{D}_{m n}\left(-\frac{\partial}{\partial x} \ln \tau\right)
\end{aligned}
$$

\section{References}

[1] H. Weyl, The theory of groups and quantum mechanics, (Dover, New York, 1931)

[2] E. Wigner, Phys. Rev. 40 (1932) 749

[3] J. E. Moyal, Proc. Cambridge Phil. Soc. 45 (1949) 99

[4] R. Kubo, J. Phys. Soc. Jpn. 19 (1964) 2127

[5] F. Bayen, M. Flato, A. Lichnerowicz, and D. Sternheimer, Ann. Phys. 111 (1978) 61, 111

[6] F. A. Berezin, Sov. Phys. Usp. 23 (1980) 763

[7] M. Hillery, R. F. O’Connell, M. O. Scully, and E. P. Wigner, Phys. Rept. 106 (1984) 121

[8] K. Sogo and Y. Fujimoto, Physica A 163 (1990) 820

[9] E. Gozzi and M. Reuter, Int. J. Mod. Phys. A9 (1994) 2191, 5801

[10] T. A. Osborn and F. H. Molzahn, Ann. Phys. 241 (1995) 79

[11] R. Hirota, J. Phys. Soc. Jpn. 50 (1981) 3785

[12] T. Miwa, Proc. Jpn. Acad. 58A (1982) 9; E. Date, M. Jimbo, and T. Miwa, J. Phys. Soc. Jpn. 51 (1982) 4116, 4125

[13] M. Sato, RIMS Kokyuroku 439 (1981) 30 
[14] I. B. Frenkel and N. Yu. Reshetikhin, Commun. Math. Phys. 146 (1992) 1; M. Jimbo and T. Miwa, Algebraic Analysis of Solvable Lattice Models, CBMS Regional Conference Series in Mathematics No. 85 (Amer. Math. Soc., Providence, 1995)

[15] R. Kemmoku and S. Saito, J. Phys. A: Math. Gen. 29 (1996) 4141

[16] C. N. Pope, L. J. Romans and X. Shen, Phys. Lett. B242 (1990) 401

[17] H. H. Chen, Y. C. Lee and J. E. Lin, Physica 9D (1983) 439

[18] A. Yu. Orlov and E. I. Schulman, Lett. Math. Phys. 12 (1986) 171

[19] L. A. Dickey, Soliton Equations and Hamiltonian Systems, (World Scientific, 1991)

[20] I. M. Gel'fand and L. A. Dikii, Russian Math. Surveys 30 (1975) 77

[21] R. Kemmoku and S. Saito, J. Phys. Soc. Jpn. 65 (1996) 1881

[22] D. B. Fairlie and C. K. Zachos, Phys. Lett. B224 (1989) 101; D. B. Fairlie, P. Fletcher, and C. K. Zachos, J. Math. Phys. 31 (1990) 1088; E. Floratos, Phys. Lett. B228 (1989) 335

[23] See for instance, $\mathcal{W}$-symmetry, Advanced Series in Mathematical Physics Vol. 22, eds. P. Bouwknegt and K. Schoutens, (World Scientific, 1995)

[24] N. M. J. Woodhouse, Geometric quantization (2nd ed.), (Oxford Univ. Press, 1991)

[25] J.-L. Gervais and A. Neveu, Nucl. Phys. B209 (1982) 125

[26] E. Frenkel, Deformations of the KdV hierarchy and related soliton equations, preprint Q-alg/9511003, (1995)

[27] I. A. B. Strachan, J. Phys. A28 (1995) 1967; A Geometry for Multidimensional Integrable Systems, Univ. of Newcastle preprint (1995)

[28] Y. Kodama, Phys. Lett. A129 (1988) 223; Y. Kodama and J. Gibbons, Phys. Lett. A135 (1989) 167; I. M. Krichever, Commun. Math. Phys. 143 (1991) 415; K. Takasaki and T. Takebe, Int. J. Mod. Phys. A7 Suppl. 1B (1992) 889

[29] C. L. Mehta, J. Math. Phys. 5 (1964) 677

[30] Yu. I. Manin, J. Sov. Math. 11 (1979) 1

[31] B. Kupershmidt, Lett. Math. Phys. 20 (1990) 19

[32] A. Yu. Orlov and P. Winternitz, Loop Algebra Symmetries and Commuting Flows for the Kadomtsev-Petviashvili Hierarchy, preprint CRM-1936, (1994)

[33] L. A. Dickey, Commun. Math. Phys., 167 (1995) 227 